\title{
Effect of Gust Wind on Flow over a Wall-Mounted Fence
}

\author{
D. Bhamitipadi Suresh ${ }^{1}$, E. J. Aju ${ }^{1}$, M. J. Zaksek ${ }^{1}$, M. M. Leffingwell ${ }^{1}$, Y. Jin ${ }^{1 *}$ \\ ${ }^{1}$ Department of Mechanical Engineering, The University of Texas at Dallas, Richardson, TX 75080, USA \\ *yaqing.jin@utdallas.edu
}

\begin{abstract}
In this work, the characteristics of incoming and wake flows downstream of wall-mounted fences under wind gust were explored with wind tunnel experiments. A time-resolved particle image velocimetry was used to capture the flow dynamics across two different fence heights. The results show that during the gust period, the wake presents distinct meandering and strong flow mixing. The Probability Density Function distribution of flow velocities indicates that the mixing effect increases with the streamwise distances. Specifically, for locations above the fence top tip, the growth of streamwise distance decreases the footprint of wind gust. However, for locations lower than the fence top tip, the local wind flows exhibit stronger variations before and after wind gust with the growth of downstream distance. Overall, at the same relative streamwise and spanwise locations downstream of fences within the wake region, the higher fence better suppresses the influence of gust wind.
\end{abstract}

\section{Introduction}

Wall Mounted Fences (WMF) have been studied for more than five decades (Plate, 1971) for their unique aerodynamic properties and applications. They have been shown to offer protection against damage caused by winds (Dong et al., 2010), reducing the wake flow and thus enhancing the deposition of air borne particles such as sand or snow (Alhajraf, 2004; Basnet et al., 2015), and accelerating wind speed at high altitude to increase the wind power production (Tobin et al., 2017), among others.

In one of the earliest works on WMFs, Raine and Stevenson (1977) studied turbulent characteristics behind both solid and porous fences and they established empirical relationships between mean velocity and turbulence intensity in the wake. A wind tunnel study conducted by Judd et al. (1996) together with a large-eddy simulation by Patton et al. (1998) showed that the fluid flow accelerates in the region above the fence. Tobin et al. (2017) illustrated that this phenomenon can be effectively captured by installing WMFs upstream of wind turbines to increase power production by at least 10\%. Kim and Lee (2001) deployed Particle Tracking Velocimetry to study turbulent shear flow upstream of WMF. Their results show that the separation point detaches and moves upstream of fence surface causing the streamline curvature to increase near the fence. More recent experimental investigation by Tobin and Chamorro (2018) found that wakemoment coefficient of WMF appeared to depend strongly on surface roughness and change with the cosine of incident angle. It, however, did not appear to change with aspect ratios (fence span length/height) of 10 or higher.

Aside from normal flow conditions, sudden intermittent or persistent increases in velocities, also known as gusts, have long been known for altering the fluid wake and change aerodynamic loading of various structures (Hayashi, 1992; Gromke and Ruck, 2018; Letson et al., 2019). In natural environments, complex topographic terrain of earth acts as a precursor of local rapid increase in velocity in atmospheric boundary layer (ABL) (Wagenbrenner et al., 2016). Hayashi (1992) investigated turbulent wind flow in ABL and found that effective mixing of flow occurs at peak gust due to efficient transport of downward momentum and that momentum flux dictates gust development. Study by Gromke and Ruck(2018) highlights the effects of gust on damage patterns in forest and it illustrates that downstream vortex behind a row of trees (can be thought of as a WMF) deflects high momentum fluid from above which increases wind loading on subsequent rows. Recent experiments by Letson et al. (2019) analyzed properties of gust wind using Doppler lidar and sonic anemometer to best describe its behavior at wind turbine heights. Their probability distributions of gust 
parameters had better agreement between the two modes of measurement for wind coherence functions on vertical displacements than on horizontal displacements.

Despite the efforts reviewed above, our understanding of how gust winds affect the wake flows of WMFs remains very limited. In this work, wind tunnel experiments with state-of-the-art particle image velocimetry (PIV) were conducted to study the incoming and wake flows of WMFs of two heights impinged by wind gusts. This study reveals the wake mixing mechanisms for structures under rapid variation of incoming flow velocities, and highlights the heterogenous wake dynamics in response to the wind gusts across various streamwise and vertical locations. Experiment set up details are discussed in Sec. II, results are illustrated in Sec. III, main conclusions and future scope are summarized in Sec. IV.

\section{Experimental Setup}

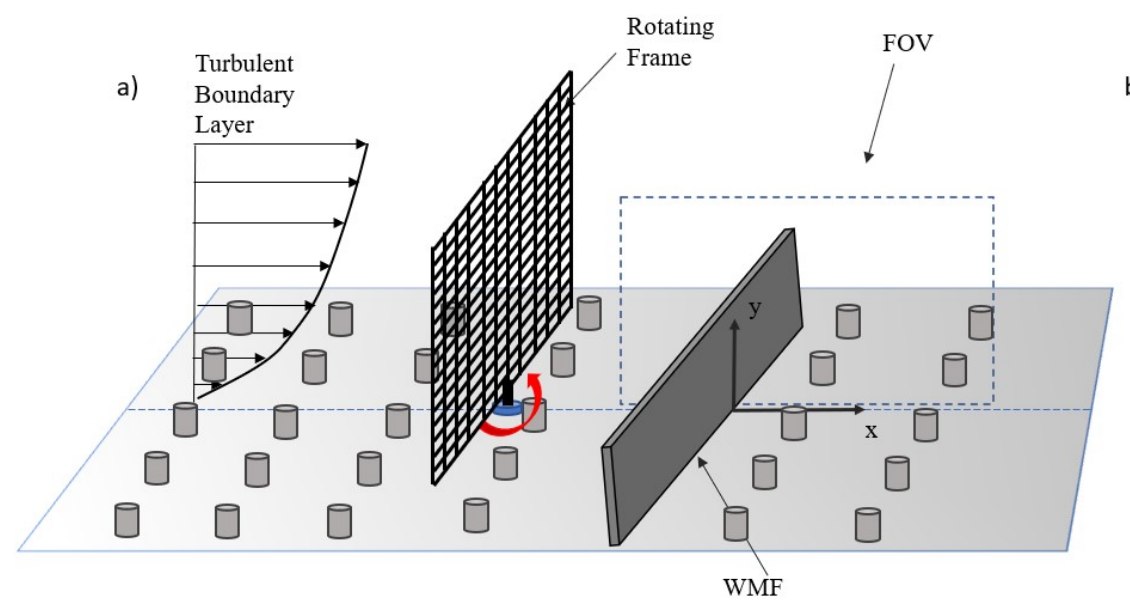

b)

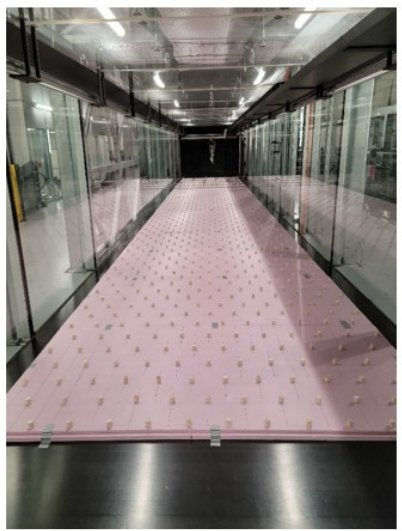

Figure 1: a) Schematic of the experimental setup; b) photograph of boundary layer roughness elements in BLAST.

Wake characteristics over two WMFs were studied in Boundary Layer and Subsonic Tunnel (BLAST) in The University of Texas at Dallas. BLAST has a testing section of $30 \mathrm{~m}$ length, $2.8 \mathrm{~m}$ width and $2.1 \mathrm{~m}$ height. The fences were mimicked by flat acrylic plates with heights $h_{1}=h$ (hereafter as low fence) and $h_{2}=2 h$ (hereafter as high fence), where $h=10 \mathrm{~cm}$, and shared the same span of $30 \mathrm{~cm}$ and thickness of 4 $\mathrm{mm}$. Both fences were vertically mounted on the bottom wall of BLAST. In this work, surface roughness with short circular cylinders of $1.5 \mathrm{~cm}$ diameter and $2.5 \mathrm{~cm}$ height every $0.25 \mathrm{~m}$ was placed along the test section to develop the turbulent boundary layer incoming flows (see figure 1b). Before the wind gust was initiated, the time-averaged incoming flow velocity at the top-tip of low and high fences $\left(U_{\text {tip }}\right)$ were $2.2 \mathrm{~m} / \mathrm{s}$ and $2.3 \mathrm{~m} / \mathrm{s}$. Details of the non-dimensional incoming velocities and turbulence intensity are detailed in figure 2.

A wooden square lattice mesh which covers the span of WMF, as shown in figure 1a, was used to block the incoming flow before the occurrence of gust. The mesh is $0.4 \mathrm{~m}$ length and $0.4 \mathrm{~m}$ width and composed with 23 horizonal and vertical wooden sticks, resulting in a porosity of $63 \%$. The lattice mesh was mounted on a computer-controlled servo motor from its base. After the incoming flow reached the state-steady with sufficient time, the motor was rotated $90^{\circ}$ within $2 \mathrm{~s}$, where the mesh became parallel to the flow. This resulted in the sudden variation of local blockage and therefore produced wind gusts. The influence of gust wind can be clearly visualized in the streamwise velocity distributions shown in figure 2c-e, where the time-averaged incoming velocity increased $\sim 23 \%$ after the gust at both $y=h$ and $y=2 h$.

To well characterize the influence of gust wind on wake flows, a time-resolved planar PIV system from TSI was implemented to measure the flow statistics. A Phantom VEO440 camera with 4 MP resolution was used to capture the flow at a frequency of $200 \mathrm{~Hz}$ for $15 \mathrm{~s}$ (3000 image pairs). The camera covered a Field of View (FOV) of $491 \times 307 \mathrm{~mm}^{2}$, corresponding to $x / h \in[-1.3,3.4]$ and $y / h \in[0.35,2.9]$ (the origin of the coordinate was set at the base of the fence and FOV was set on the center of span). The air flow was seeded with soap bubbles of mean diameter $15 \mu \mathrm{m}$, and the FOV was illuminated with a $1 \mathrm{~mm}$ thick laser 
sheet from a high-speed laser. The captured image pairs were then interrogated using Insight $4 \mathrm{G}$ software from TSI. With a multi-pass image processing, the final interrogation window size was $24 \times 24$ pixels ${ }^{2}$ with $50 \%$ overlap, resulting in a final vector grid spacing of $\Delta x=\Delta y=2.3 \mathrm{~mm}$. Overall, the uncertainty for the identification of seeding particle locations was approximately 0.1 pixel, leading to the uncertainty of flow velocity measurement of $\sim 1.4 \%$ considering the bulk particle displacement of $\sim 7$ pixels between two successive images.
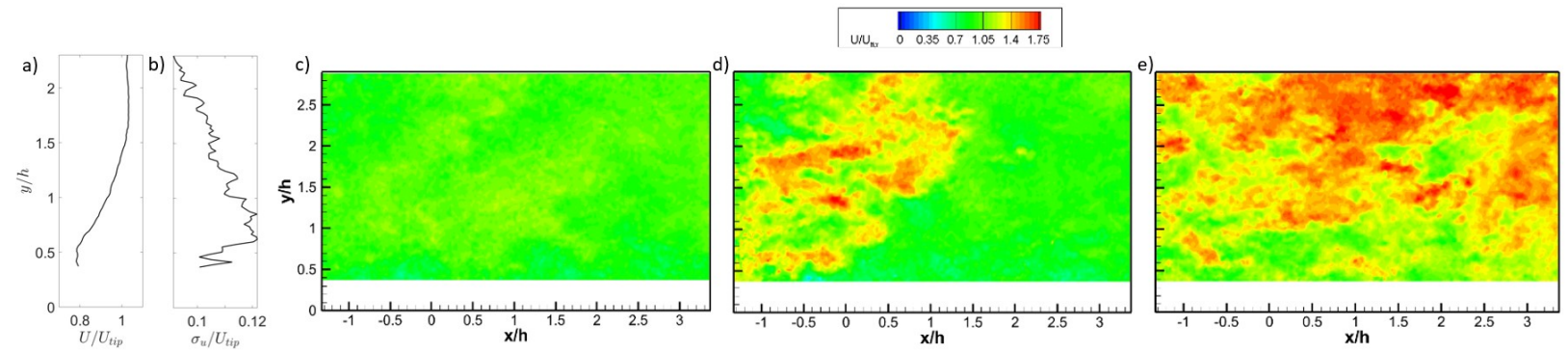

Figure 2: a): time-averaged streamwise velocity profile of incoming boundary layer flow; b) is same as a) but for the turbulence intensity. c-e) are representative instantaneous velocity distributions within the FOV before, during and after wind gust.
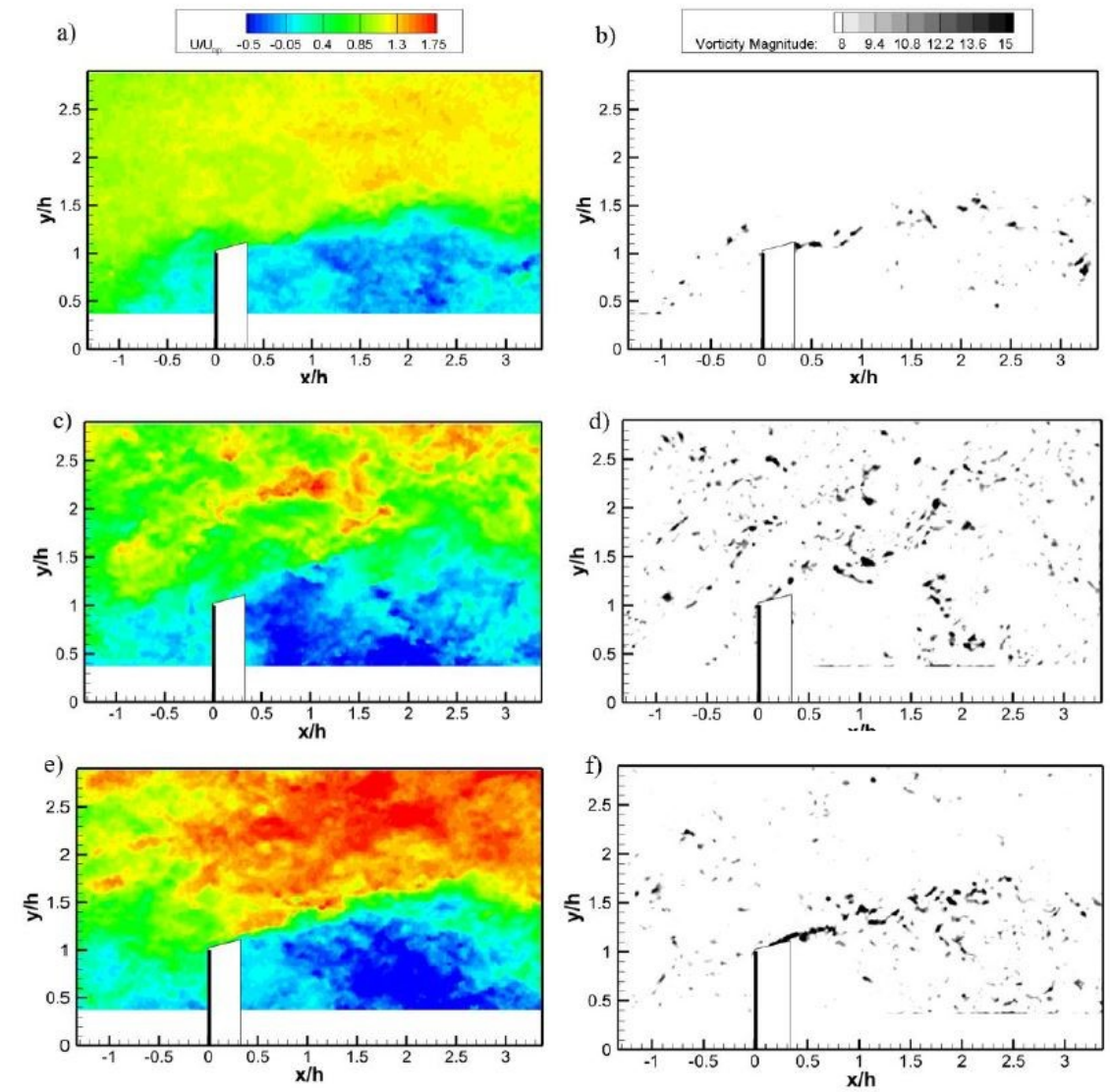

Figure 3: Streamwise velocity distribution over low fence, (a) before gust, (c) during gust, (e) after gust. Figures (b), (d), (f) are corresponding vorticity contours. 


\section{Result and Discussion}

The influences of wind gust on the wake flows of fences are first illustrated via the normalized streamwise velocity distribution and wake vorticity magnitude in figure 3. Before the wind gust was initiated, the wake flow presents clear velocity shear near the top tip of the fence, which produces the distinctive Kelvin-Helmholtz instability (figure 3a,b). The occurrence of wind gust leads to strong disturbance to the flow. As illustrated in figure $3 \mathrm{c}$, the velocity magnitude in both wake region (i.e., $y / h<1$ ) and upper region (i.e. $y / h>1$ ) increased during the wind gust. During this process, the wake flow also presented clear "meandering' dynamics, where the Kelvin-Helmholtz instability no long concentrates along $y / h \approx 1$. It is worth pointing out that due to the sudden variation of local wind velocities, strong flow mixing occurred during the wind gust, where vortical structures across different scales are observed in the incoming flow and upper region (figure 3d). The characteristics of flow reached a new steady-state after the wind gust passed (figure $3 e, f)$. Although the velocity magnitude significantly increased compared to the instant before gust, the location of velocity shear remains similar as those observed in figure 3a. This resulted in the similar distribution of Kelvin-Helmholtz instability but with overall stronger magnitude compared to the instants before gust. These phenomena were also observed for the high fence illustrated in figure 4. The time series of wind
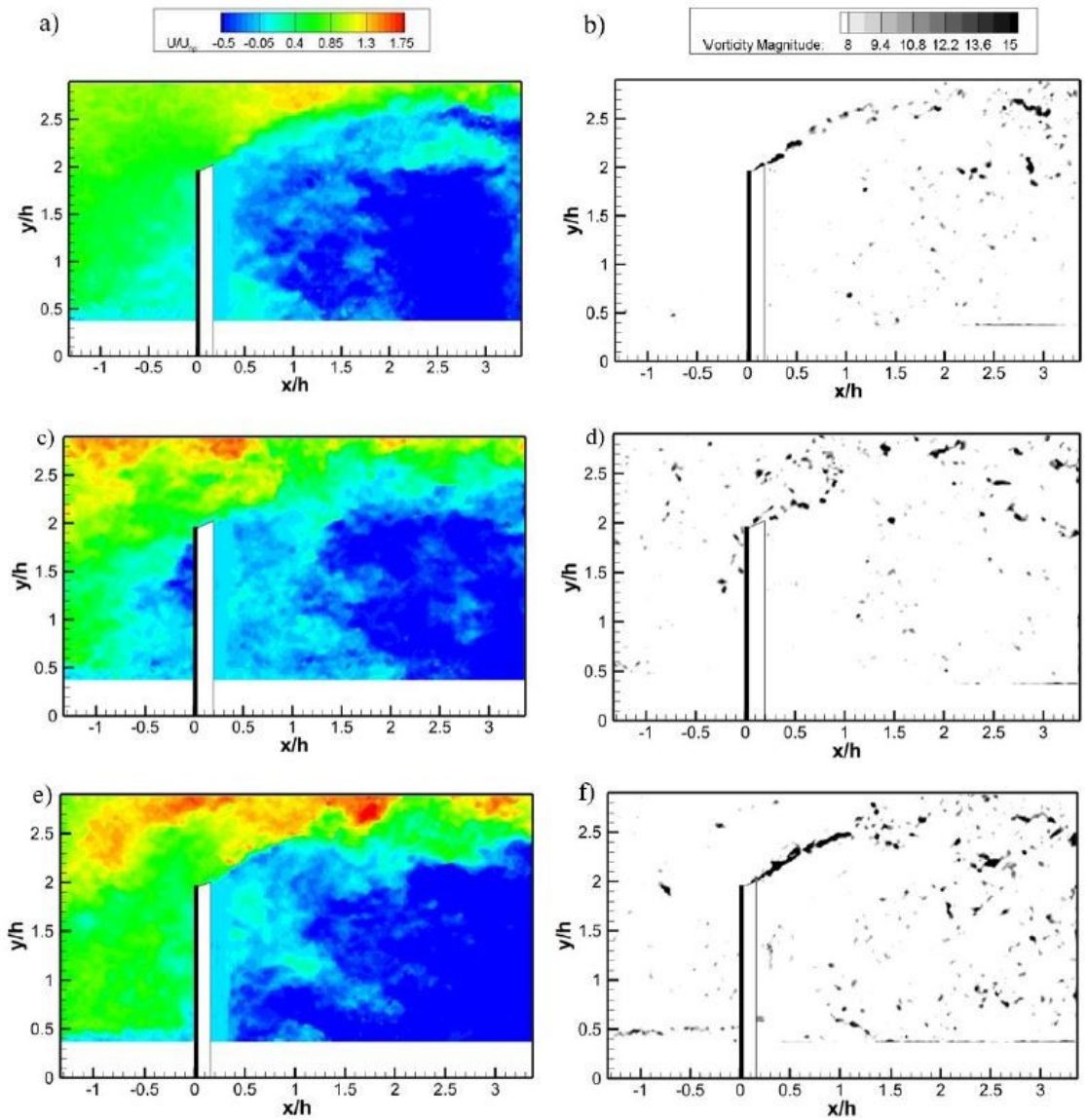

Figure 4: Streamwise velocity distribution over high fence, (a) before gust, (c) during gust, (e) after gust. Figures (b), (d), (f) are corresponding vorticity contours.

velocities within the upper region across various streamwise locations are illustrated in figure 5a. Overall, the wind velocities present sudden increase right after the occurrence of gust wind. To further highlight the variation of local flow velocities, the corresponding Probability Density Function (PDF) distribution of wind velocities are presented in figure $5 \mathrm{~b}-\mathrm{d}$. Specifically, in the very near wake region $(x / h=1)$, the PDF presents two distinctive peaks, corresponding to the flow velocities before and after the wind gust (figure 5 b). Interestingly, the second peak representing the higher wind velocity after gust gradually weakened with the growth of streamwise distances (figure $5 \mathrm{c}, \mathrm{d}$ ). Note that the height of wake region gradually expands 

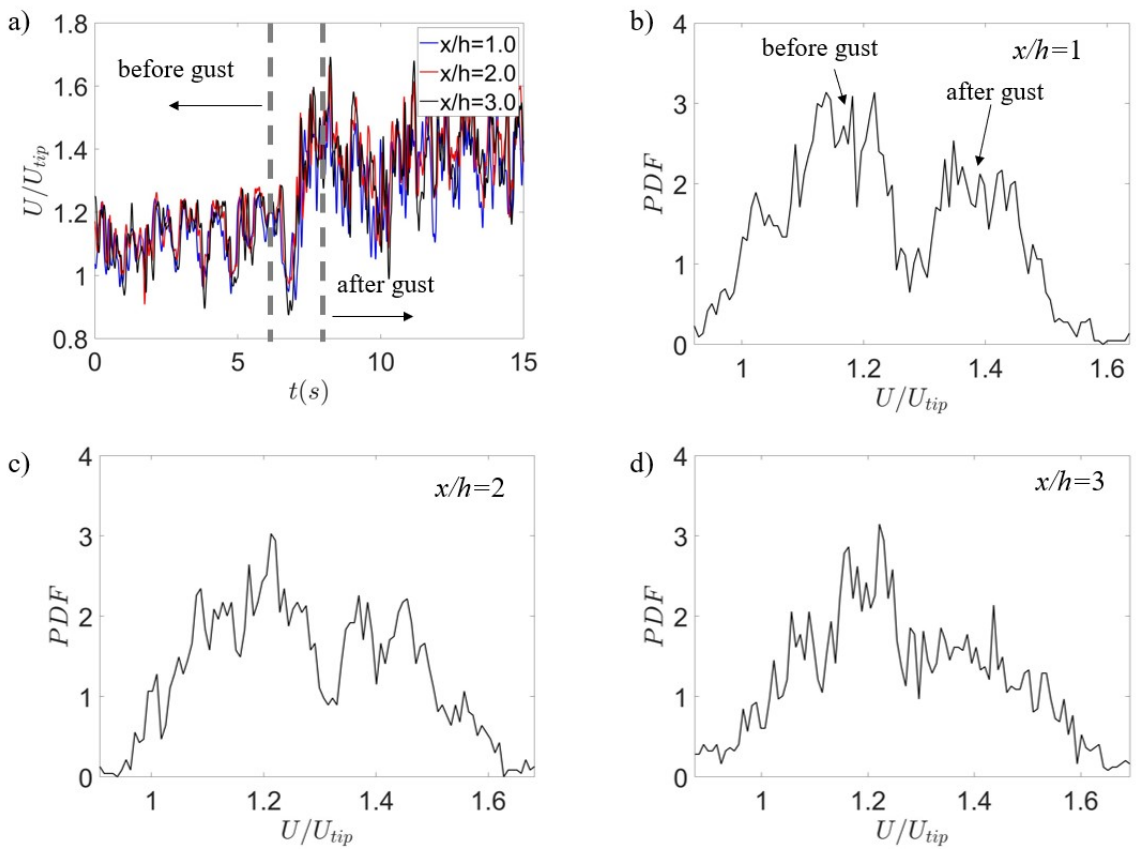

Figure 5: a) Representative time series of streamwise velocity $U / U_{\text {tip }}$ for low fence at $y / h=2.5$. (b-d) are the corresponding PDF distribution of normalized streamwise velocities.
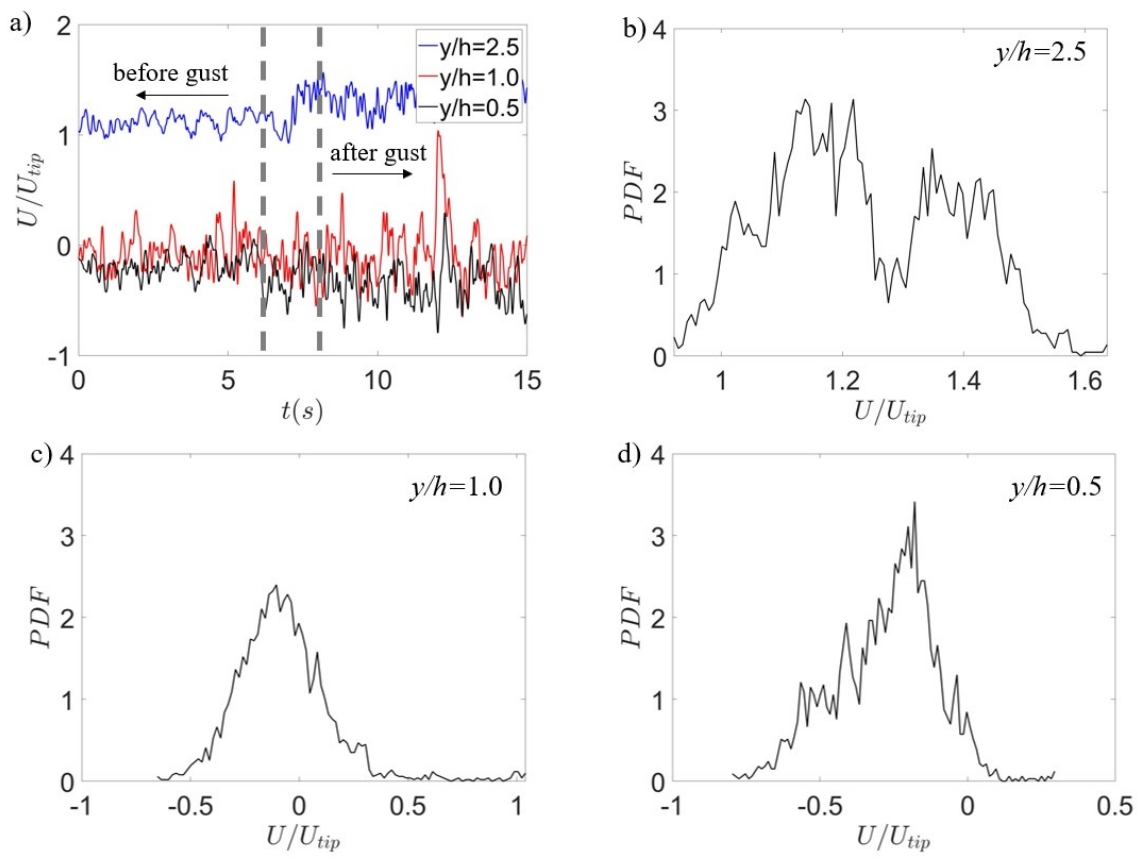

Figure 6: a) Representative time series of streamwise velocity $U / U_{\text {tip }}$ for low fence at $x / h=1$. (b-d) are the corresponding PDF distribution of normalized streamwise velocities.

along the streamwise distance (see figure 3); therefore, the wind velocities located further downstream experience stronger mixing effects with the wake flows. This results in broader local wind velocity distributions and suppresses the intensity of secondary peak initiated by wind gust. Similar to the cases along streamwise direction, the wind gust also produces heterogeneous influences on local velocity fluctuations along the vertical direction. While wind velocities within the upper region present distinct variation after the gust with 
dual peaks in PDF distribution, this is not the case within the wake flow (figure 6); here, the PDF distribution of wind velocities presents a sole peak for $y / h \leq 1$. It is worth pointing out that the wake velocities exhibit much stronger fluctuations than those within the upper region. This results in a wide range of overlap between the wind velocities before and after the wind gust, thus suppresses the appearance of 'dual peaks' in the PDF distribution.

Finally, to highlight the influence of fence height on wake velocity variations during wind gust, the PDF distributions of streamwise flow velocity before and after wind gust at the same relative locations downstream of both fences are illustrated in figure 7 and 8 . Here, all locations are at the half fence height but with different streamwise distances. Overall, the PDF distribution shifts towards the negative values of $U / U_{\text {tip }}$ after the occurrence of wind gust, which is induced by the stronger backward flow (i.e., $U<0$ ) as illustrated in figure 3 and 4 . Regardless of fence height, the gust wind produces little influence on wind velocities within the very near wake regions (figure 7a and 8a). In particular, the PDF distribution of wake velocities is nearly identical before and after wind gust for the high fence at $x / h=1$. With the growth of streamwise distances, the flow mixing between wake and upper regions becomes stronger and more rapid, where more footprint of wind gust enters the wake region thus resulting in larger differences of flow velocities before and after gust. Such differences are in general more distinctive for the lower fence, indicating that within the same turbulent boundary layer, the wake flows of structures with smaller size are more sensitive to the influence of wind gusts.
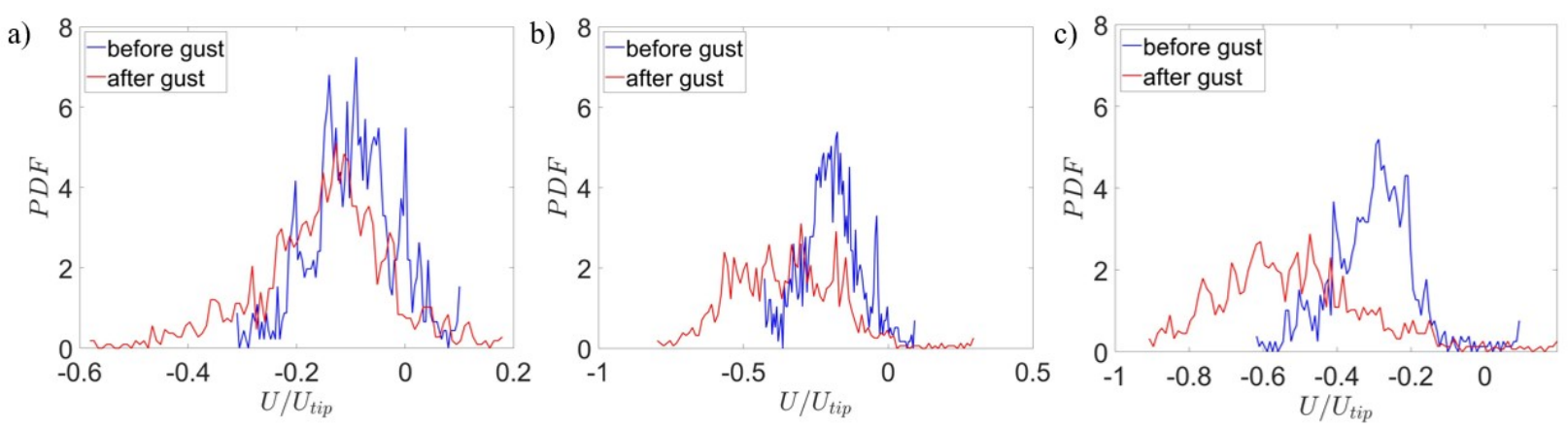

Figure 7: PDF distribution of normalized wake velocity downstream of low fence at $y / h=0.5$ and a): $x / h=0.5 ; \mathrm{b}): x / h=1 ; \mathrm{c}): x / h=1.5$.
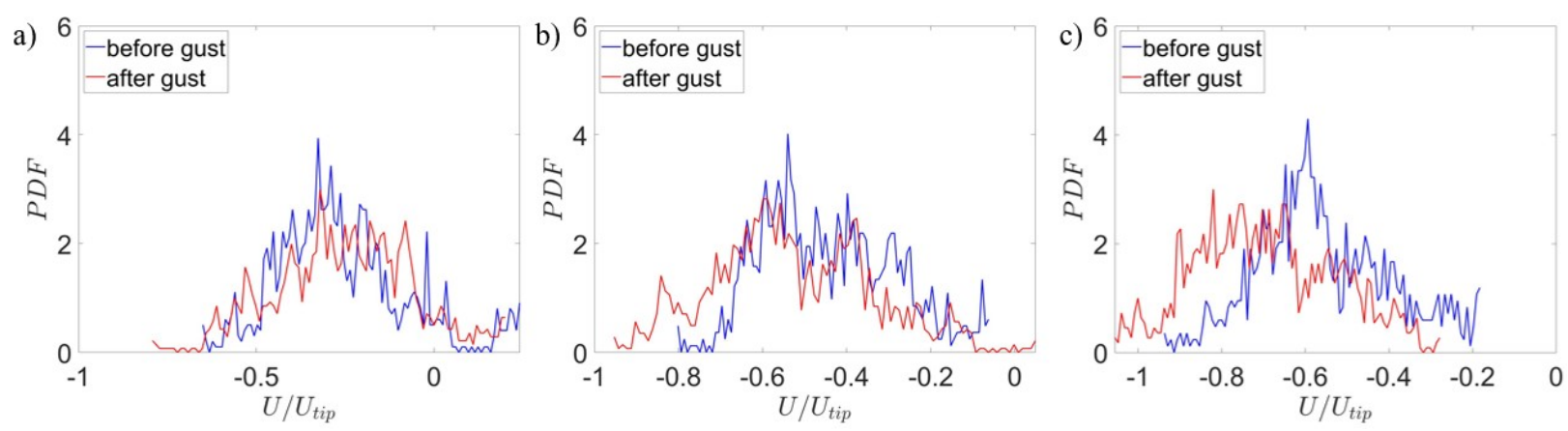

Figure 8: PDF distribution of normalized wake velocity downstream of high fence at $y / h=1$ and a): $x / h=$ $1 ; \mathrm{b}): x / h=2 ; \mathrm{c}): x / h=3$.

\section{Conclusions}

The occurrence of wind gust leads to distinctive flow mixing and wake meandering downstream of wallmounted fences. For locations above the fence height within the very near wake, the PDF distribution of wind velocities reveals two clear peaks corresponding to velocities before and after gust. However, this 
phenomenon is gradually diminishing with the growth of streamwise distances due to the stronger mixing effects, as well as the decrease of vertical distances due to the higher local flow fluctuations. In general, for both fences within the very near wake region, the local flow characteristics present little variations before and after wind gust. Our future work will explore the influence of wind gust with various intensities, and its impact on more complex structures such as wind turbines and urban buildings.

\section{Acknowledgements}

This work was supported by the Department of Mechanical Engineering, the University of Texas at Dallas, as part of the start-up package of Dr. Y.J.

\section{References}

Alhajraf S (2004) Computational fluid dynamic modeling of drifting particles at porous fences. Environmental Modelling \& Software 19:163-170

Basnet K, Constantinescu G, Muste M, and Ho H (2015) Method to assess efficiency and improve design of snow fences. Journal of Engineering Mechanics 141:04014136

Dong Z, Luo W, Qian G, Lu P, and Wang H (2010) A wind tunnel simulation of the turbulence fields behind upright porous wind fences. Journal of Arid Environments 74:193-207

Gromke C and Ruck B (2018) On wind forces in the forest-edge region during extreme-gust passages and their implications for damage patterns. Boundary-Layer Meteorology 168:269-288

Hayashi T (1992) Gust and downward momentum transport in the atmospheric surface layer. Boundarylayer meteorology 58:33-49

Judd MJ, Raupach MR, and Finnigan JJ (1996) A wind tunnel study of turbulent flow around single and multiple windbreaks, part i: velocity fields. Boundary-Layer Meteorology 80:127-165

Kim HB and Lee SJ (2001) Time-resolved velocity field measurements of separated flow in front of a vertical fence. Experiments in fluids 31:249-257

Letson F, Barthelmie RJ, Hu W, and Pryor SC (2019) Characterizing wind gusts in complex terrain. Atmospheric Chemistry and Physics 19:3797-3819

Patton EG, Shaw RH, Judd MJ, and Raupach MR (1998) Large-eddy simulation of windbreak flow. Boundary-Layer Meteorology 87:275-307

Plate EJ (1971) The aerodynamics of shelter belts. Agricultural Meteorology 8:203-222

Raine JK and Stevenson DC (1977) Wind protection by model fences in a simulated atmospheric boundary layer. Journal of Wind Engineering and Industrial Aerodynamics 2:159-180

Tobin N and Chamorro LP (2018) Wakes behind surface-mounted obstacles: Impact of aspect ratio, incident angle, and surface roughness. Physical Review Fluids 3:033801

Tobin N, Hamed AM, and Chamorro LP (2017) Fractional flow speed-up from porous windbreaks for enhanced wind-turbine power. Boundary-Layer Meteorology 163:253-271

Wagenbrenner NS, Forthofer JM, Lamb BK, Shannon KS, and Butler BW (2016) Downscaling surface wind predictions from numerical weather prediction models in complex terrain with windninja. Atmospheric Chemistry and Physics 16:5229-5241 